\title{
A NEW METHOD. FOR THE PREPARATION OF BRONCHOPULMONARY CASTS
}

\author{
BY \\ D. H. TOMPSETT \\ From the Royal College of Surgeons of England, London
}

(RECEIVED FOR PUBLICATION OCTOBER 5, 1951)

In the preparation of corrosion casts of the bronchial tree four factors must be considered which are not met with when ordinary arterial or venous injections are made. (1) The material used must be sufficiently strong to be self-supporting, and unaffected by the reagent used to destroy the lung tissue. (2) As the branches of the tree terminate in alveoli which constitute about four-fifths of the expanded lung, it is necessary to employ some technique which prevents the injection filling these, since, if this happens, it will be impossible to remove the alveolar tissue. (3) Since the lungs collapse when the thorax is opened, a bronchial cast made from such lungs would have little value. Therefore, unless it is possible to make the cast in situ, a technique must be employed whereby the lungs are expanded to something like their original size while the injection is being made. (4) The residual air in the lungs may cause various complications. This air cannot be removed simply by squeezing the lungs, and a special technique must be employed.

Three distinct methods of injection have been employed, each of which has advantages and drawbacks. (1) The use of a substance which has a relatively high melting point, and sets on cooling; Wood's metal, with a melting point of about $71^{\circ} \mathrm{C}$., is generally considered the most suitable substance of this type. (2) The use of a plastic dissolved in a volatile solvent, which evaporates leaving a solid cast ; a saturated solution of polyvinyl chloride in acetone has been recommended for this method. (3) The use of a resin which sets under the influence of a catalyst into a hard substance, for example, one of the Marco resins.

The chief advantage of the Wood's metal and polyvinyl chloride methods is that they are self-limiting, provided that conditions are correctly adjusted. That is to say, the injection becomes solid before reaching the alveoli, so that the cast can be prepared by maceration without resorting to tedious pruning. It is, however, found in practice that chance plays an important role in deciding the degree of success which can be achieved by these two methods. The chief disadvantage is that unless the lungs can be injected in situ a comparatively elaborate vacuum chamber is necessary so that they may be injected in the expanded condition without distorting the general contour. A further objection to Wood's metal is that it cannot be coloured to differentiate the vessels and bronchi.

Many variations of technique have been described, using Wood's metal and polyvinyl chloride. Details of the former are given by Smyth (1949) and of the latter by Liebow, Hales, Lindskog, and Bloomer (1947).

When using resins which are set by means of a catalyst, the principal difficulty is the tendency of the resin to flow into the alveoli before it sets. If a fairly complete 
cast is required it is apparently not possible to prevent this altogether. Consequently the cast cannot be prepared by maceration alone, but some pruning is always necessary.

The method described here was developed from the results of 20 experiments, using Marco resin $26 \mathrm{C}$. In these experiments first dog, then sheep, and finally human material was used. By this method it is possible to prepare every time an almost perfect cast of the bronchial tree in the expanded condition, together with the arteries and veins, provided that the operator has the skill and patience which it demands. The final pruning may take up to 10 days to complete if the vessels as well as the bronchi are filled and it is desired to preserve the finer branches, but if the bronchi only are filled, the pruning can usually be completed in three days.

The advantages are that no elaborate apparatus is required, all the work is done after the lungs have been removed from the body, the lungs are injected in the fully expanded condition, with the lobes arranged in the physiological position, and a comparatively perfect specimen is always obtained. The disadvantages are the labour involved, and the considerable skill required to obtain the best results.

\section{MATERIAL}

To obtain the best results healthy lungs must be obtained in which the blood has not yet clotted. They should be removed undamaged from the body, together with the heart and trachea. It has been found that post-mortem lungs after two days in a refrigerator are quite satisfactory.

\section{Procedure}

Short pieces of glass tubing should be fixed into the trachea, the pulmonary artery, and the left atrium. These should be flanged to prevent them slipping out, and tied very firmly in place with string. Anything finer than string tends to cut into the tissues.

The lungs are placed in a basin of water, and the blood washed out by running cold water from the tap into the trachea and allowing it to escape via the arteries and veins after diffusing through the walls of the alveoli. The apparatus used both for washing out the blood and removing the air is shown in Fig. 1. When it is used for washing out the blood, clamps are placed at $\mathbf{A}$ and $\mathrm{C}$ so that the water can only flow into the lungs.

To remove the air from the lungs they are alternately inflated with carbon dioxide and evacuated. This process is repeated about 10 times. While the lungs are being inflated a clamp is fixed at $\mathbf{B}$, and moved to $\mathrm{C}$ during evacuation. Then cold water is run through the trachea for a few minutes until the carbon dioxide which remains in the lungs has been dissolved. If any air remains in the lungs, this process should be repeated. About 5 litres of $70 \%$ spirit are next poured into the trachea to displace the water, and the lungs placed in a $70 \%$ spirit tank to fix them.

When this stage has been reached, the lungs may be kept for several weeks in spirit without deterioration, but the following stages must be completed in one day.

The lungs are transferred to a large water bath, and the spirit washed out by running water from the tap alternately through the trachea and vessels for two hours. This sometimes results in air from the tap water accumulating in the lungs. If necessary repeat the process of removing the air with carbon dioxide. When all is ready for the injection of the resin the temperature of the water bath is raised to $33^{\circ} \mathrm{C}$. and water at this temperature is run through the trachea for a short time to warm the lungs. The water bath is maintained at this temperature until the injection of the resin has been completed. 


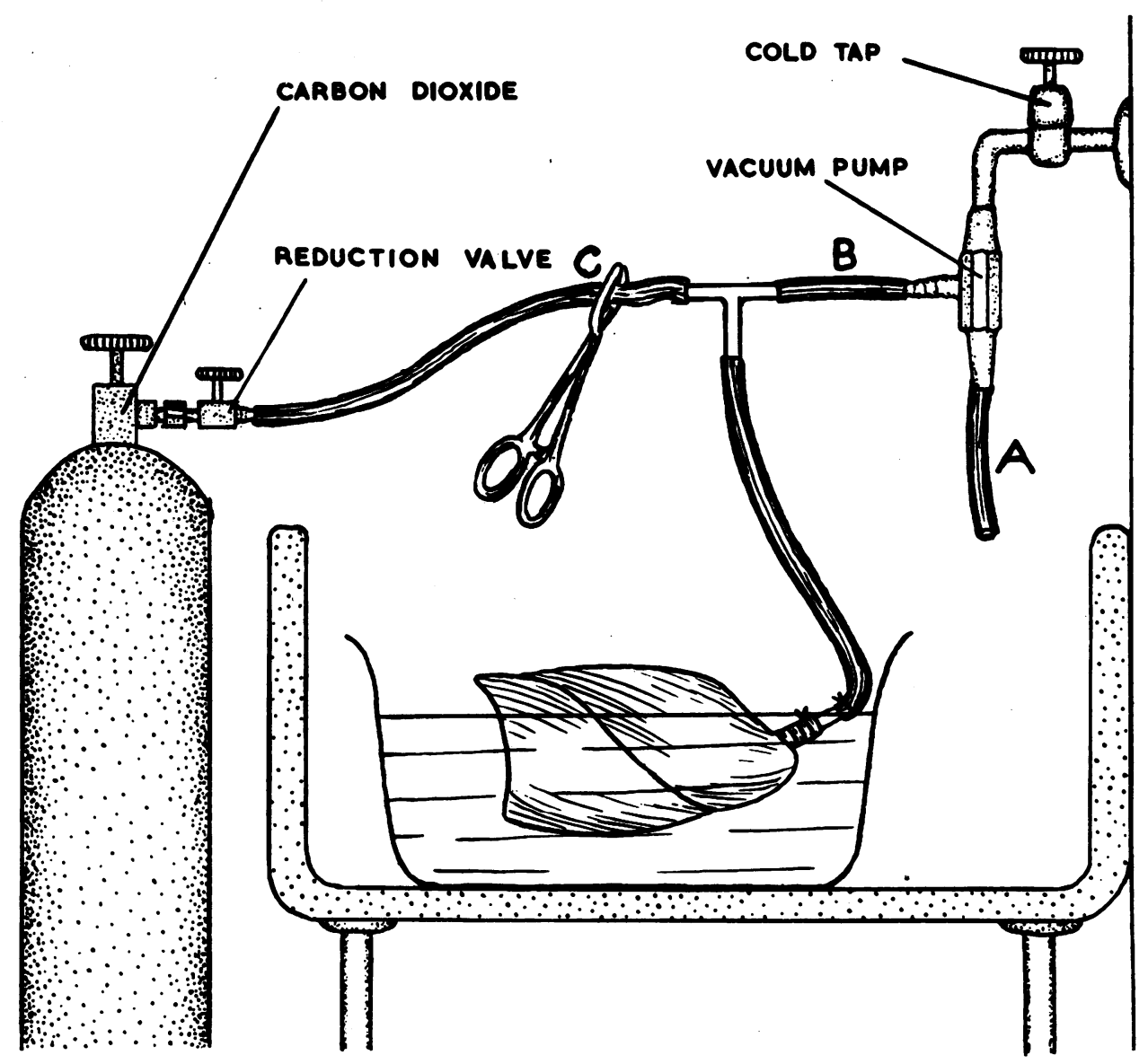

Fig. 1.-Apparatus for washing out blood and removing carbon dioxide from the lungs.

The lungs are then placed on a plaster mould (Fig. 2) shaped roughly in the form of the posterior thoracic wall and gelatin (see Appendix) at $33^{\circ} \mathrm{C}$. is poured into the trachea until the lungs are quite turgid and fully expanded. For a large specimen about 5 litres will be required, as some gelatin diffuses into the water bath.

The gelling point of gelatin is about $28^{\circ} \mathrm{C}$. It is essential that it shall be used at a temperature only a few degrees above $28^{\circ} \mathrm{C}$. because the object of the gelatin is to maintain the lungs in an expanded state, and at the same time to prevent too much resin from entering the alveoli. This is only achieved if the gelatin is in a viscous state, which it only assumes at temperatures close to its gelling point.

The specimen is now arranged in its natural position, and all the apparatus for the injection of the resin (Fig. 3) placed so that the rubber tubes to the vessels do not press against the lungs; 6-in. funnels can be used to contain the resin. These should have the lower two-thirds of their tapering stalks cut off, as the relatively narrow bore at the bottom retards the flow of the viscous resin. The rubber tubing which is used to connect the funnels to the lungs should have an internal diameter of not less than $\frac{3}{8}$ in. 
FIG. 2.-Plaster mould on which lungs are placed during injection.

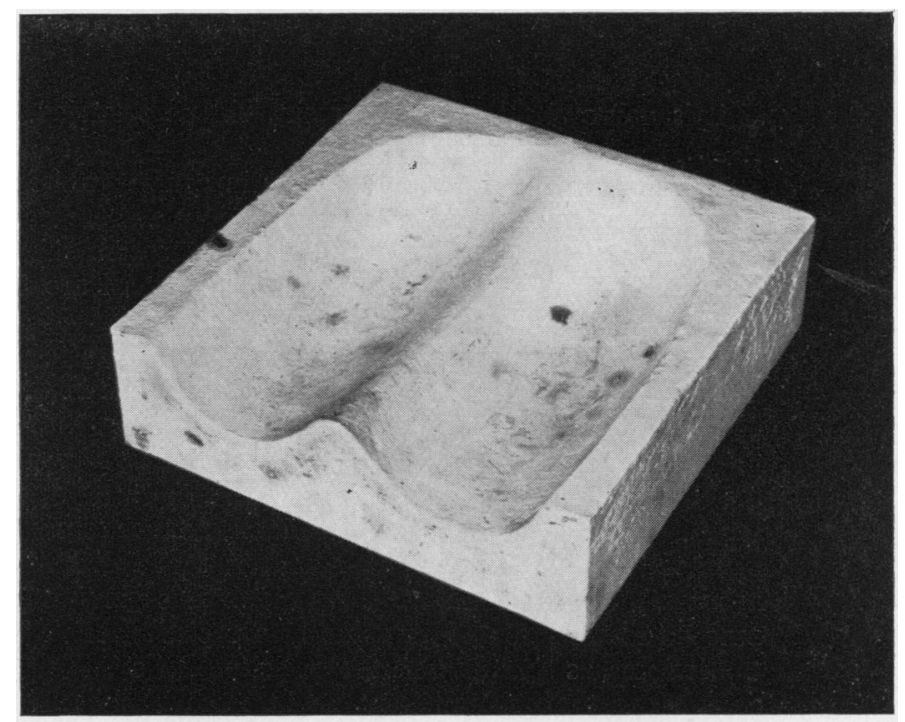

FIG. 3.-Apparatus just before the injection of resin. Stand and clamps holding the funnels are not shown.

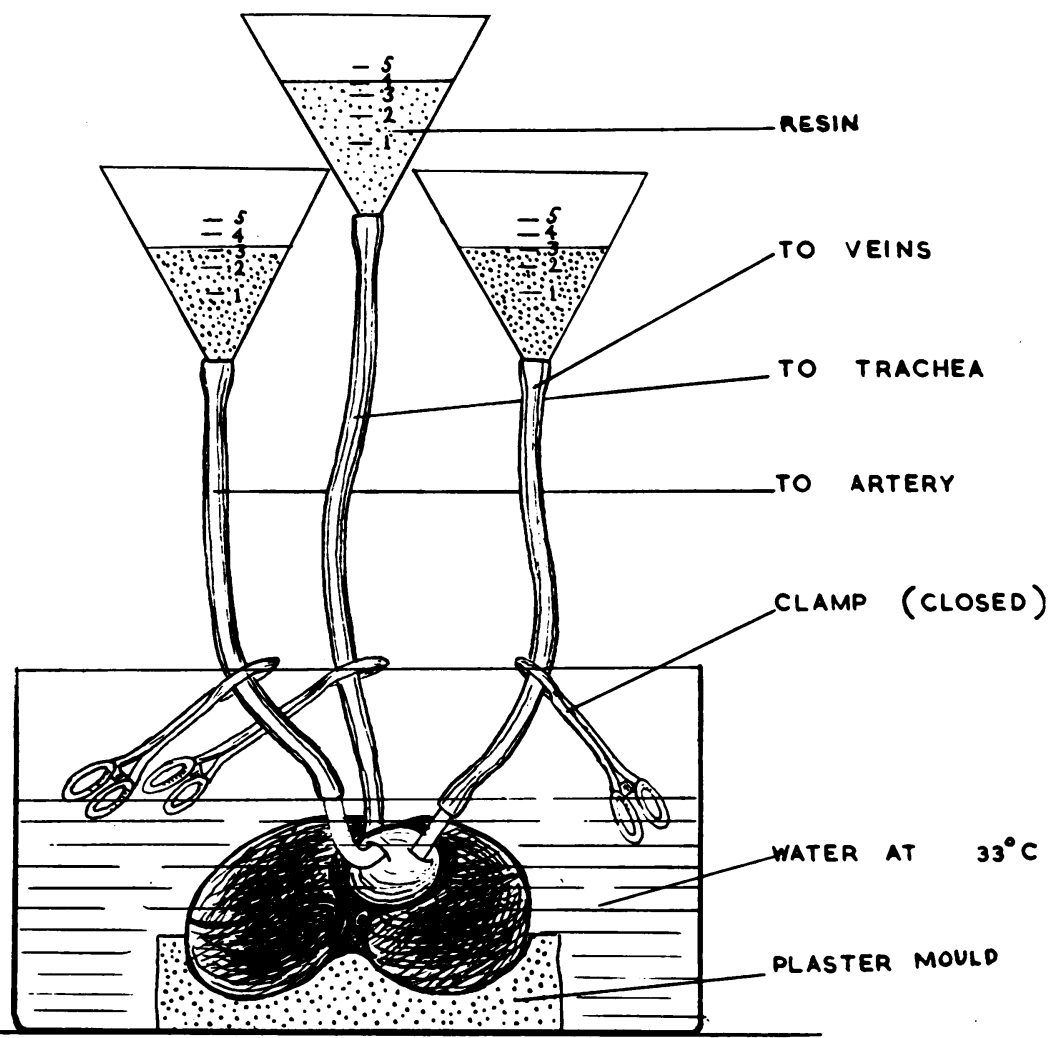


If it is narrower the flow of resin will be retarded. The funnels should be held about 18 in. above the lungs.

Syphon out as much of the gelatin as the total amount of resin which it is estimated will be run in (the exact amount of gelatin removed is not important, since gelatin displaced by the resin will diffuse out through the pleura fairly readily). For a large specimen, if the vessels as well as the bronchi are to be filled, $800 \mathrm{ml}$. are about right. If only the bronchi are to be filled, then about $400 \mathrm{ml}$. would be sufficient.

Each of the rubber tubes from the specimen is disconnected in turn, and the appropriate resin (prepared according to instructions in the Appendix) poured into the funnel, holding the end of the tube in such a position that the resin flows just to the end of it to avoid trapping air in it. Then apply a clamp to prevent the flow of resin and reattach the tube to the specimen.

All three clamps are released simultaneously. The resin flows quite rapidly into both the vessels and bronchi. After about two minutes it will cease to flow into the arteries and veins. Note how much has flowed into these (approximately the same amount flows into the veins as into the arteries), and allow twice as much to flow into the trachea as has entered the arteries. In a large specimen about $200 \mathrm{ml}$. flow into the arteries, so that $400 \mathrm{ml}$. are run into the trachea.

The successful injection of the vessels presents no difficulty, but the injection of the bronchi is a more delicate operation. Although the resin fills all the bronchi evenly when it is first run in, if the flow is stopped when $400 \mathrm{ml}$. have entered, the resin in the bronchi continues to flow into the alveoli, so that in time the uppermost bronchi will become empty. For although the gelatin retards the flow of resin into the alveoli, it does not prevent it altogether. It is therefore necessary to compensate for this flow of resin within the lungs. Immediately $400 \mathrm{ml}$. have entered the bronchi, the tube through which the resin is flowing is clamped, and a large quantity of ice is added to the water bath. This will set the gelatin at the surface of the specimen, and so prevent the resin from continuing to flow into the peripheral alveoli. But until the resin gells, about $25 \mathrm{ml}$. should be run in every two minutes to top up the resin in the bronchi. Failure to do this will result in an incomplete cast.

As the work of pruning away the surplus resin from the specimen after maceration depends on how much surplus resin was originally run in, it is desirable to time the injection so that the minimum amount of topping up is necessary. But it is unwise to cut this time too close, as the gelling time does not appear to be quite constant. It is wise to allow about six minutes' margin if a mixture which gells in 20 minutes is used.

When the resin has gelled in all the funnels, cut through the rubber tubes and the resin they contain to disconnect the funnels (Fig. 4). The surplus resin in the funnels and rubber tubing can be removed after setting is completed, as it contracts slightly on setting. (If the bronchi only are being injected, see Appendix for modifications of procedure.)

The specimen is left undisturbed in the water bath for at least a week for the resin to harden. Then the heart is dissected away, and the three short glass tubes which are filled with resin are removed by cracking the glass. The ends of resin projecting from the pulmonary artery and the left atrium are sawn off with a gigli saw. The resin projecting from the end of the trachea is left in situ so that the specimen can be held by this during the subsequent pruning. At this stage the lungs should be handled with great care, as their weight while filled with gelatin is such that the resin may be broken if they are not carefully supported when they are lifted out of the water.

The specimen is now washed in running water at a temperature of not more than $40^{\circ} \mathrm{C}$. for two days to allow as much gelatin as possible to diffuse out. After cooling the specimen by substituting cold water for hot, in order to harden the resin, it is lifted carefully out of the water and placed in a bath of concentrated hydrochloric acid in 
which it should be completely immersed. The lungs are left in this bath for 24 hours. It is essential that the specimen shall not be placed in the acid until nine days after the injection. If it is placed in acid sooner the incompletely hardened resin may be attacked by the acid and the cast made so brittle that it falls to pieces.

The specimen is placed in a large basin of cold water, and the macerated tissue washed away with a jet of hot water. The hot water makes the resin very flexible and enables the macerated tissue at the centre of the lungs to be washed away more readily. If necessary, the specimen may be returned to the acid bath for a further 24 hours. When the washing is completed it will be found that part of the tree is already displayed, but there are sure to be areas where the injection has spread to the alveoli which obscure the main branches of the bronchi. The surplus resin must be removed from these areas by pruning. Fig. 5 is a photograph of a cast of the bronchi before it has been pruned. In this specimen the vessels have not been filled.

Finally the end of resin projecting beyond the trachea is attached vertically to a clamp, at the same time supporting as much of the weight of the specimen as possible by suspending it in a basin of water (Fig. 6). The surplus resin is most easily removed by pruning the specimen with a pair of curved scissors, and tearing it away with curved forceps. During this operation the specimen must be kept wet, as the resin becomes very brittle when it dries. If necessary a jet of hot water may be directed on to the part which is being pruned.

If any important branches are broken off they should be stuck

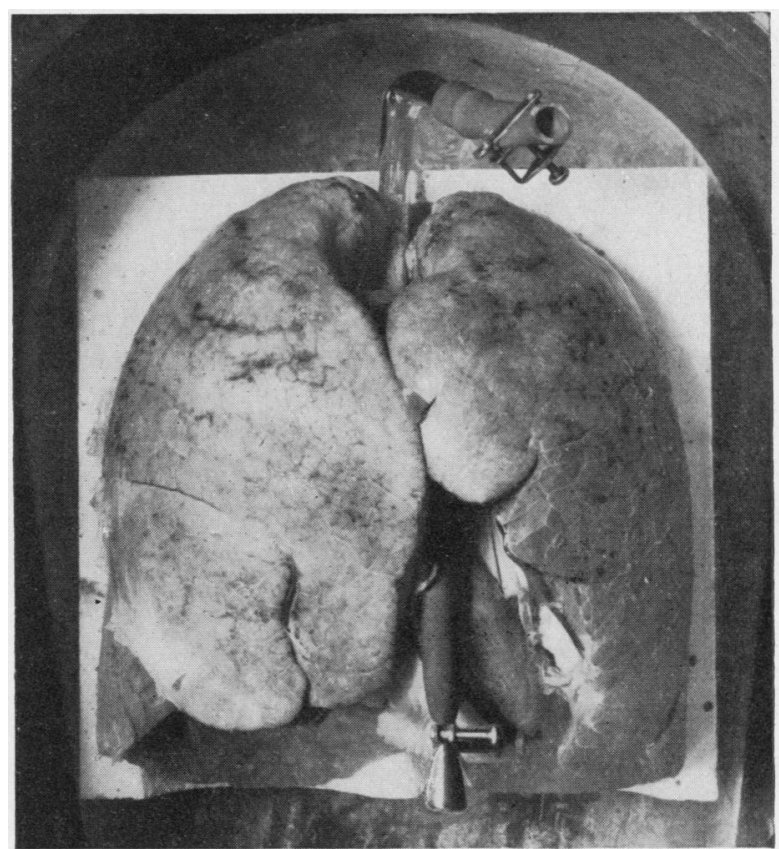

FIG. 4.-Lungs immediately after injection of bronchi and arteries. $\times \frac{1}{3}$ on again with a little Marco resin $28 \mathrm{C}$ (see Appendix) which makes a join as strong as the original resin and quite invisible.

If the cast has become distorted during pruning, it can usually be restored to its original form by immersing it in hot water, as when it is softened in this way the resin tends to return to its original shape. If it is desired to alter the form of the cast, this can be done by bending the resin after it has been softened by a jet of steam, and fixing it in the new position by cooling with a jet of cold water.

If it is found that the walls of the bronchi and vessels are very difficult to remove from the cast because they have become impregnated with resin, this is a result of injecting the resin before all traces of spirit have been removed from the lungs.

Although the colours of the resin may show up brilliantly while the specimen is wet, when it dries the whole surface will assume a whitish colour. The colours can 


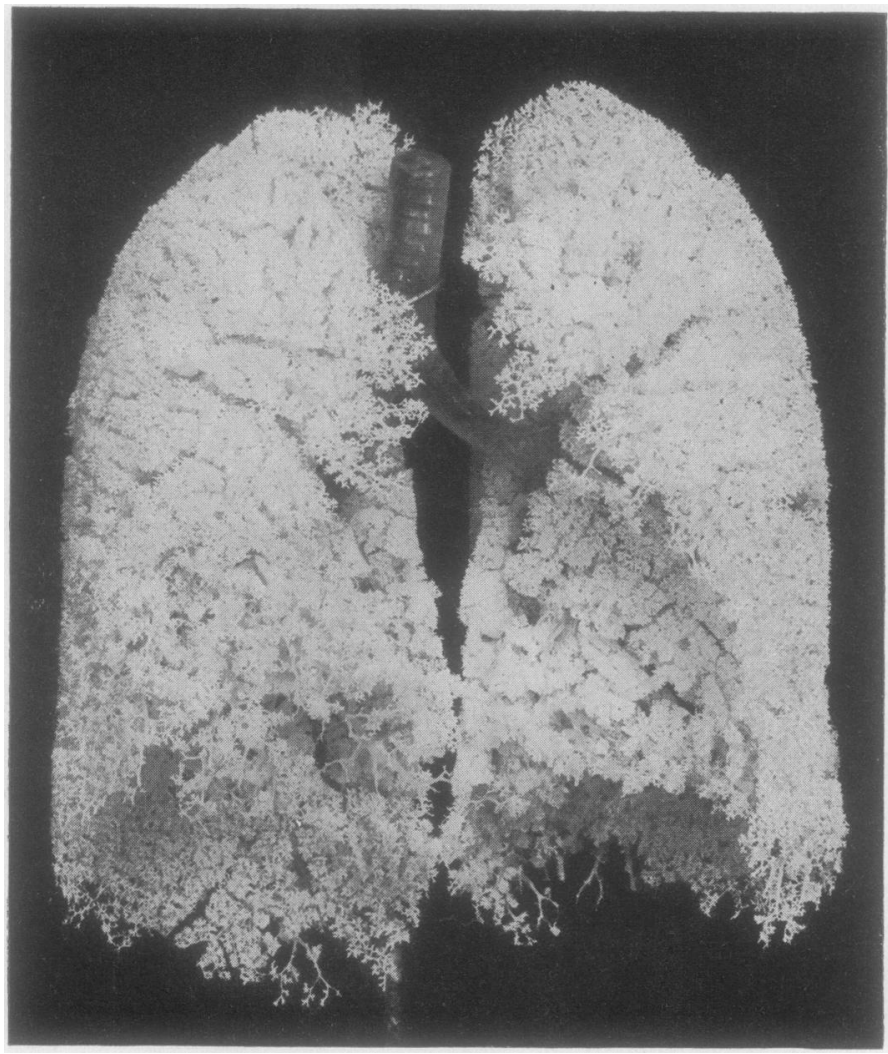

Fig. 5.-Cast of adult human bronchial tree, after maceration, but before pruning. $\times \frac{1}{3}$

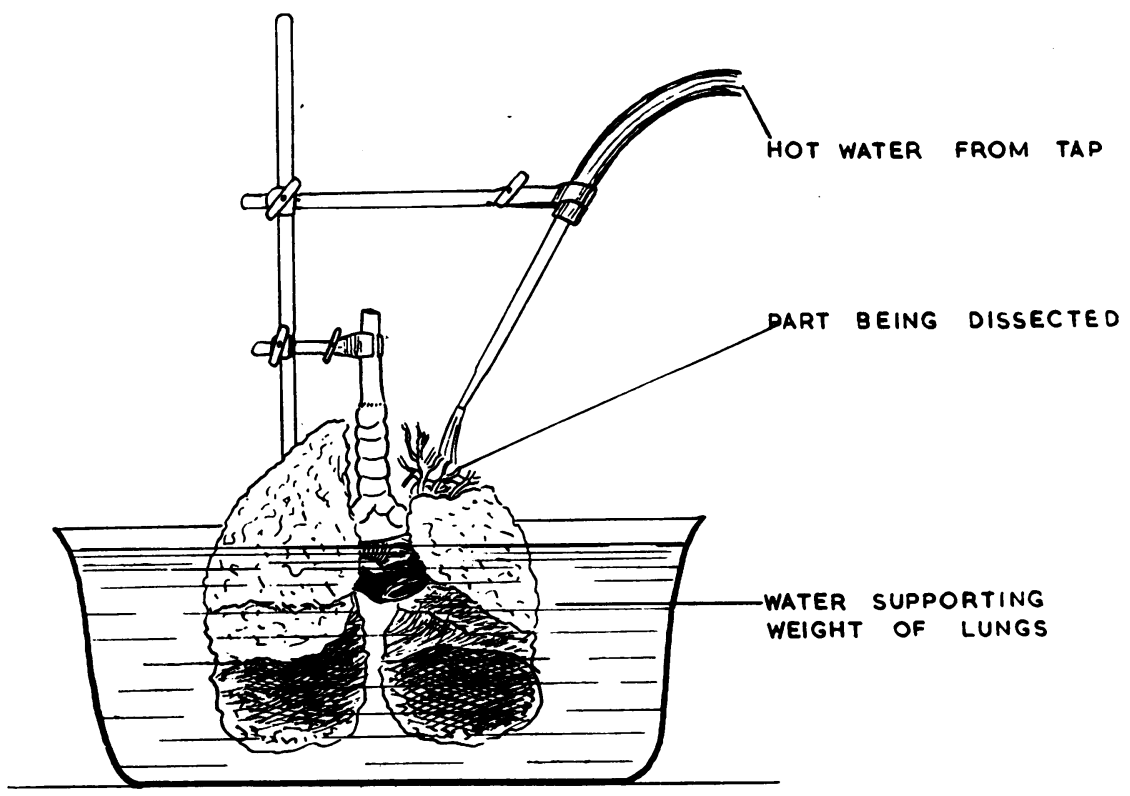

FIG. 6.-Pruning of macerated lungs. 
PREPARATION OF BRONCHOPULMONARY CASTS

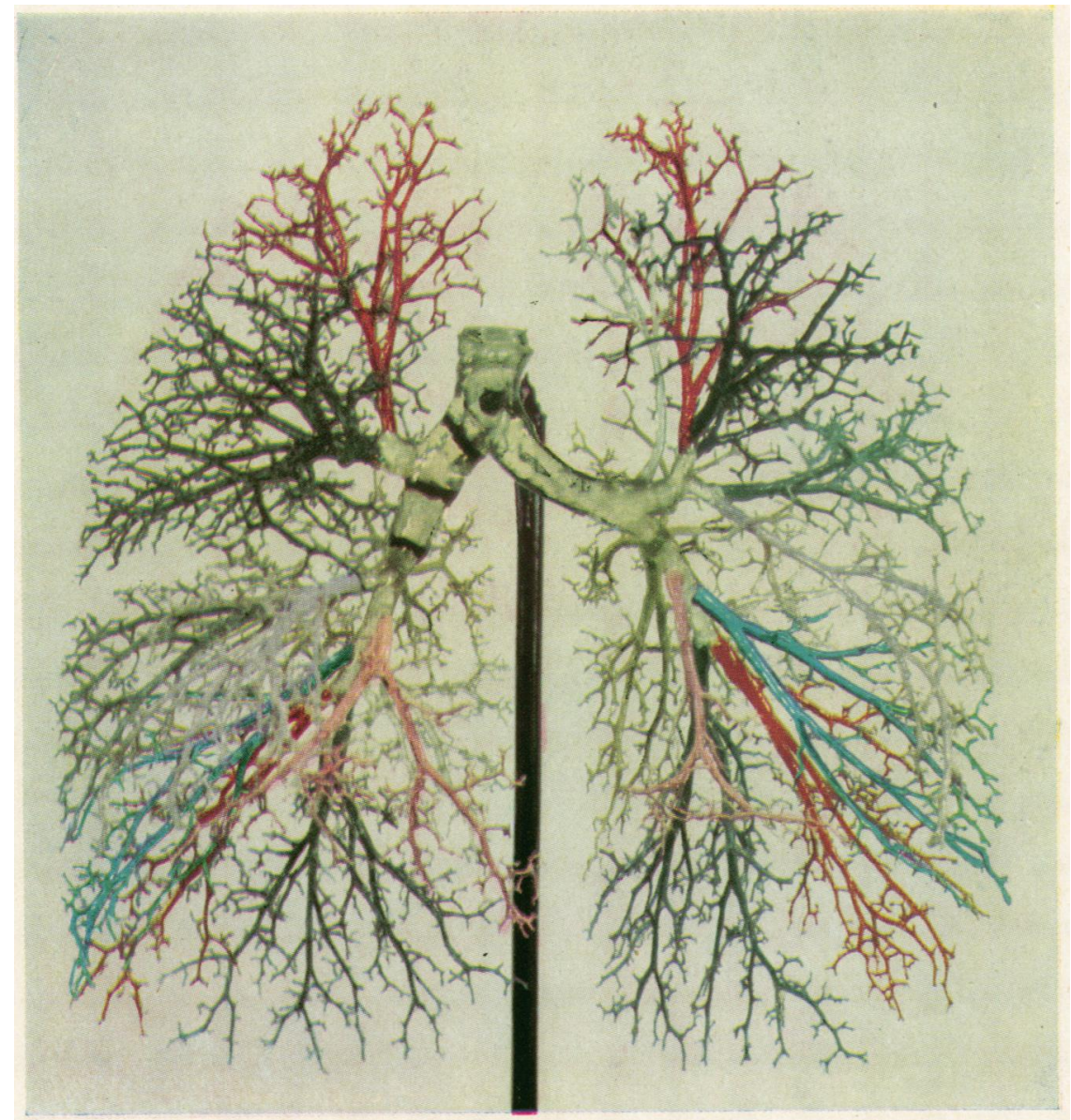

Fig. 8. Cast of human bronchial tree in which the bronchi have been painted. $\times \frac{4}{9}$ 
D. H. TOMPSETT

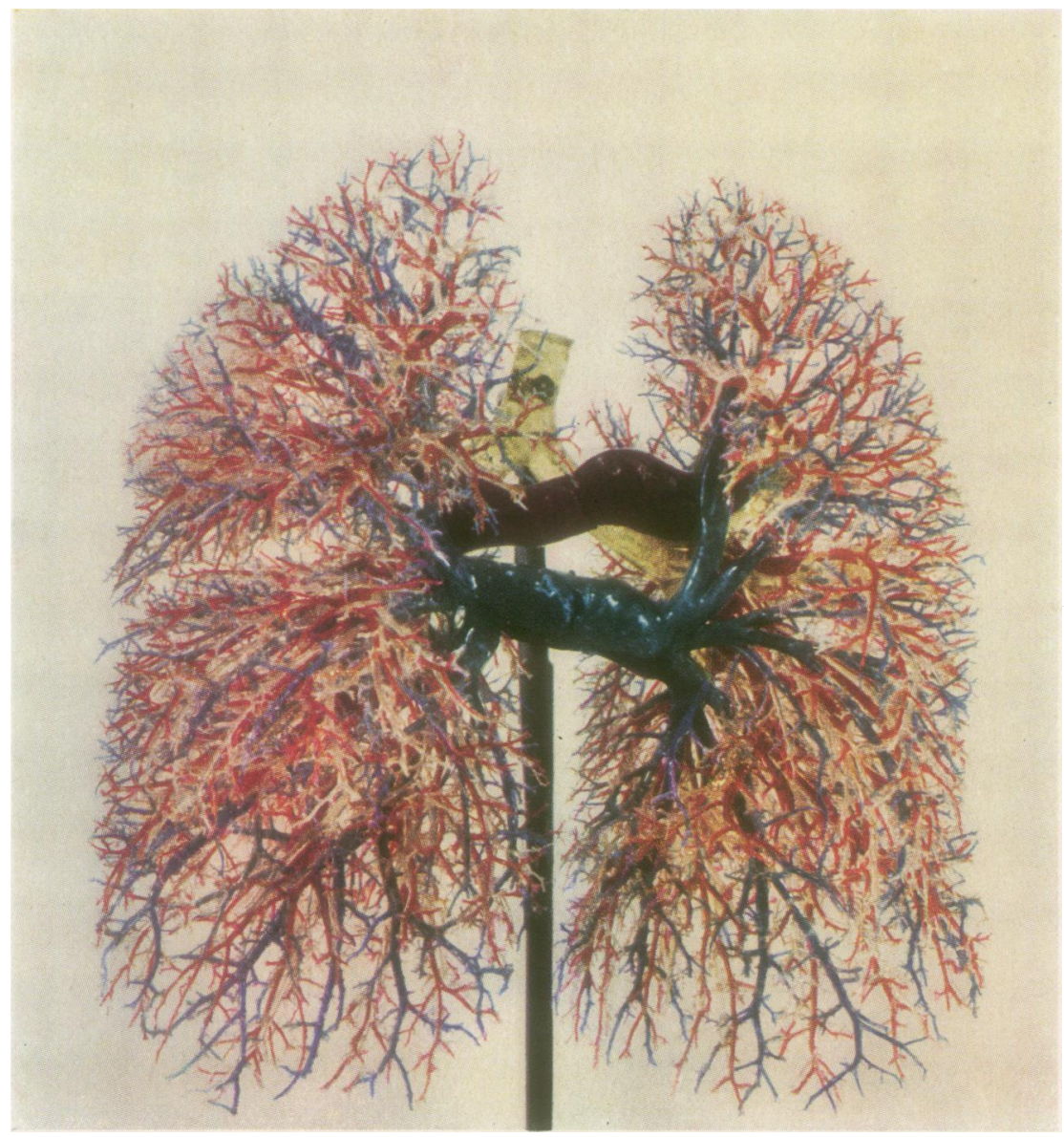

Fig. 9. Cast of human bronchial tree with arteries and veins. $\times \frac{4}{9}$ 
be permanently restored by spraying the specimen with a solution of Marco resin $28 \mathrm{C}$ (see Appendix).

Fig. 7 illustrates a method of mounting the specimens.

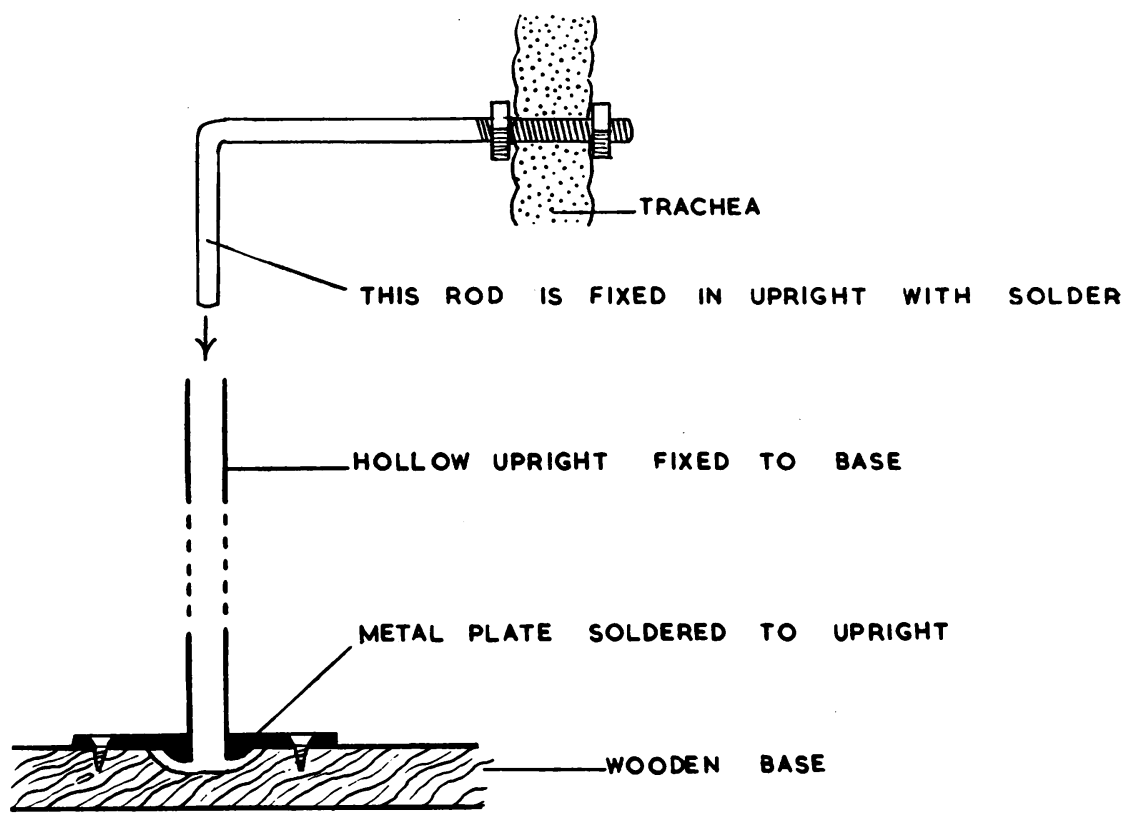

FIG. 7.-Method of mounting finished specimen.

Photographs of two casts made from relatively large adult human lungs by the method described here are shown in Figs. 8 and 9.* Fig. 8 is a photograph of a bronchial tree in which the principal segments have been subsequently differentiated with paints. After the painting was completed, the whole cast was sprayed with Marco resin $28 \mathrm{C}$ so that the paints can never, flake off. Fig. 9 is a photograph of an adult bronchial tree with the pulmonary arteries and veins injected. This specimen is almost perfect, and the photograph shows that the lungs were fully expanded at the time of injection, and also the extent to which the natural contours have been preserved.

\section{SUMMARY}

A new method of preparing bronchopulmonary casts is described. First the blood is washed out of the vessels. Then the air is removed as completely as possible, and the lungs fixed in spirit. After washing the spirit out with water, the lungs are filled with gelatin, so that they are expanded. Next the resin is run in, and after this has set the tissue is removed by maceration in concentrated hydrochloric acid. Then the surplus resin in the alveoli is pruned away. Finally the original brightness of the specimen is restored by spraying it with a solution of resin.

* The specimens illustrated in Figs. 8 and 9 and others prepared by this method can be seen in the museum of the Royal College of Surgeons of England. 


\section{A P P E N D I X}

The following are further details of techniques and materials.

Gelatin Solution.-Gelatin solution is prepared by soaking leaves of gelatin overnight in cold water, straining the soaked leaves, and melting them over a water bath.

Marco Resin Technique.-For the injection of the bronchial tree and vessels Marco resin $26 \mathrm{C}$ was used.*

When the room temperature was about $20^{\circ} \mathrm{C}$. the following formula was used :

$\begin{array}{lllllrl}\text { Marco resin 26 C } & \ldots & \ldots & \ldots & 100 \mathrm{~g} . \\ \text { Monomer C } & \ldots & \ldots & \ldots & \ldots & 15 & \mathrm{~g} . \\ \text { Catalyst H C H } & \ldots & \ldots & \ldots & \ldots & 4 \mathrm{~g} .\end{array}$

If the room temperature is about $25^{\circ} \mathrm{C}$., then only $12 \mathrm{~g}$. of monomer $\mathrm{C}$ should be used, as an increase of room temperature reduces the viscosity of the resin.

For red resin Marco pigment crimson lake M. 23 was added; for the blue, Marco pigment M. 21.

The catalyst is dissolved in the monomer, aided by gentle warming. Then enough pigment is added to give a sufficiently intense colour, and the monomer is mixed with the resin. The above mixture, with or without colour, is stable for at least six hours.

Immediately before use the following amount of accelerator $\mathrm{E}$ is added per $100 \mathrm{~g}$. of original resin, and very thoroughly stirred in :

$$
\begin{array}{lllll}
\text { For colourless resin } & \ldots & \ldots & \ldots & 2.0 \mathrm{ml} \\
\text { For red resin } & \ldots & \ldots & \ldots & 1.5 \mathrm{ml} \\
\text { For blue resin } & \ldots & \ldots & \ldots & 1.0 \mathrm{ml}
\end{array}
$$

The reason why a different amount of accelerator is added according to the colour of the resin is that the colouring matter affects the gelling time.

The above mixtures gell in approximately 25 minutes, but it is essential to make a test to establish the exact gelling time of each resin mixture, as this varies according to room temperature and the age of the materials used. If it is desired to increase the gelling time less accelerator should be used. It is unlikely that, even when varying amounts of accelerator are added to the different coloured resins, they will all take exactly the same time to gell. But it is desirable that the three resin mixtures shall gell as nearly as possible simultaneously when the injection has been made. To achieve this result the exact time at which the accelerator is added to each of the resin mixtures should be related to the time which each test sample took to gell. For example, if the red resin takes five minutes longer to gell than the other two when the test is made, then, before the injection is made, the accelerator should be added to the red resin five minutes before it is added to the blue and colourless resins.

The test to establish the gelling time of each mixture is made in the following way. If a mixture containing 7 units of the formula, $100 \mathrm{~g}$. resin, $15 \mathrm{~g}$. monomer, $4 \mathrm{~g}$. catalyst, has been made up (the weight of colouring matter is so relatively. small that it can be ignored), then 1 unit of this mixture (i.e. $119 \mathrm{~g}$. of it) should be decanted into a beaker, and 1 unit (i.e. $2 \mathrm{~g}$., $1.5 \mathrm{~g}$. or $1.0 \mathrm{~g}$. according to the colour of the resin) of accelerator added. The time which each of these test samples takes to gell is usually within four minutes of the time taken by the rest of the mixture. But when adding the accelerator to the rest of the resin, it should be remembered that there are now only 6 units left.

Until gelling is about to take place there is no indication of any change taking place in the resin. But gelling is rapid, and within two minutes of beginning the resin is com-

* Ootainable from Scott Bader Ltd., 109 Kingsway, London, W.C.2. 
pletely gelatinous. Considerable heat is evolved during gelling if a large bulk of resin is involved, as for example, if a considerable amount of resin remains in the funnels. But the final hardening is a more gradual process and continues for about a week at normal room temperature.

After the accelerator has been stirred in, the mixture should be allowed to stand for three minutes while the larger bubbles rise to the surface. Small bubbles of air may be ignored.

If the bronchi only are being injected, then certain modifications of technique indicated below, designed to reduce the labour of pruning the cast, should be followed.

If possible the resin, monomer, and accelerator should be kept in a refrigerator. At room temperature the accelerator keeps for about two months, the monomer for about four months, and the resin for six, but in a refrigerator these materials will keep for at least three times as long.

Sunlight acts as a powerful accelerator, and so care must be taken to prevent sunlight falling on these materials when they are being used for injection.

Spraying Mixture.-For spraying the finished cast the following mixture was used :

$\begin{array}{lllllrr}\text { Marco resin 28 C } & \ldots & \ldots & \ldots & 50 & \mathrm{~g} . \\ \text { Monomer C } & \ldots & \ldots & \ldots & \ldots & 20 & \mathrm{~g} . \\ \text { Catalyst H C H } & \ldots & \ldots & \ldots & \ldots & 1.0 & \mathrm{~g} . \\ \text { Acetone } & \ldots & \ldots & \ldots & \ldots & 10.0 \mathrm{ml} \\ \text { Accelerator E } & \therefore & \ldots & \ldots & \ldots & 1.0 \mathrm{ml} .\end{array}$

The catalyst is dissolved in the monomer, and then mixed with the resin. The acetone and accelerator are added immediately before use, the acetone to reduce the viscosity. At normal room temperature this mixture will remain sufficiently fluid to be used in a spray for at least 30 minutes, but it does not gell for about two hours. Unlike Marco resin $26 \mathrm{C}, 28 \mathrm{C}$ sets with a hard surface in air. But as the surface treated with this mixture will not become truly hard for about a week, the specimen should be protected from dust during this period.

Provided a good quality spray is used, this mixture will give a spray with quite fine droplets. But in order to produce this spray, the rubber bulb has to be squeezed with some violence. It requires considerable effort to maintain the spray in operation for sufficient time to complete the spraying of the whole cast, as it is necessary to direct the spray from all angles if every part of the cast is to be covered. At the same time if the glass reservoir of the spray is clasped in a warm hand for long, the heat may cause premature gelling of the resin within the spray.

It is possible to devise a very simple apparatus for spraying the specimen which eliminates this manual labour. Remove the rubber bulb from the spray and replace it by rubber tubing connecting to a cylinder of carbon dioxide, to which a reducing valve has been attached. Cut the rubber tubing in half, re-rejoining it by means of a T-piece of glass tube, one end of which remains open. Tie all the rubber tube connexions firmly and allow a moderate but continuous escape of carbon dioxide from the cylinder. When it is required to use the spray, all that is necessary is to close the open end of the T-piece with a finger. The force of the spray may be regulated by adjusting the flow of carbon dioxide. A cylinder of compressed air would serve equally well, but as a cylinder of carbon dioxide is used during another stage in this technique, it will probably be found more convenient to use the same cylinder for the spraying.

After use the spray should at once be washed out very thoroughly with acetone before the resin gells. When it has set the resin is insoluble, and can only be removed by burning. 
Marco Resin $28 \mathrm{C}$. - When using Marco resin $28 \mathrm{C}$ as a cement, about $25 \mathrm{ml}$. are poured into a beaker and about $2 \mathrm{~g}$. of catalyst paste $\mathrm{H}$ added. Mix thoroughly. This mixture will keep for at least six hours.

When cement is needed, transfer a small quantity of the mixture to a shallow porcelain dish by means of a glass rod. Add a few drops of accelerator $E$ and mix thoroughly.

See that the two ends to be joined are quite dry. Apply the cement liberally and leave till it sets. This usually takes about 20 minutes, but the joint will not be really strong until the next day. Remove the surplus cement immediately after it has gelled before it gets really hard.

Cleaning off Resin.-Apparatus contaminated with resin should be cleaned by immersing it in boiling water for an hour. This will set the resin quite hard so that it can be easily removed. Resin can be removed from the hands etc. by means of a piece of cotton wool soaked in acetone.

Cast of Bronchi Only.-The description of the injection which has been given is primarily for the simultaneous injection of the bronchi, arteries, and veins. It is likely that often a cast of only the bronchial tree will be required. If this is so it is possible to make certain small modifications of technique which will reduce the amount of resin which enters the alveoli, and hence, the amount of work involved in pruning the cast after maceration. These modifications are as follows :

(1) Use a slightly more viscous mixture than that recommended above by adding only $12 \mathrm{~g}$. of monomer instead of $15 \mathrm{~g}$. to every $100 \mathrm{~g}$. resin when the temperature is about $20^{\circ} \mathrm{C}$. If the room temperature is about $25^{\circ} \mathrm{C}$. then add only $10 \mathrm{~g}$. monomer. This rather more viscous mixture is not quite so easy to handle, but will not enter the alveoli so readily.

(2) Use $3 \mathrm{ml}$. accelerator instead of $2 \mathrm{ml}$. per $100 \mathrm{~g}$. resin. This gives a mixture with a working life of about 15 minutes. This time is ample if only the bronchi are injected, and the working life is less variable than when less accelerator is used.

(3) Begin to run in the resin only five minutes before it is due to gell, instead of eight minutes before. This cuts down the amount of resin which has to be run in during the "topping up" process described above. But if the risk of premature gelling is to be avoided, the mixing of the ingredients must have been very thorough, and the test to establish the exact gelling time must have been made with the greatest care.

(4) Run in only $300 \mathrm{ml}$. instead of $400 \mathrm{ml}$. of resin for an average specimen.

If these modifications of technique are adopted, then a cast is produced in which the pruning can usually be completed in three days.

\section{REFERENCES}

Liebow, A. A., Hales, M. R., Lindskog, G. E., and Bloomer, W. E. (1947). Bull. int. Ass. med. Mus., 27, 116.

Smyth, N. P. D. (1949). Irish J. med. Sci., 278, 62. 